SLAC-PUB-10677

BABAR-PROC-04/031

hep-ex/0409006

August, 2004

\title{
Rare Hadronic B Decays
}

\author{
Liliana Teodorescu \\ Brunel University, West London \\ Uxbridge, UB8 3PH, United Kingdom \\ (from the BABAR Collaboration)
}

\begin{abstract}
A review of recent results on branching fractions and time-integrated $\mathrm{CP}$ asymmetries of rare $B_{u}$ and $B_{d}$ decays to mesonic final states from BABAR Belle and CLEO experiments is presented.
\end{abstract}

Contributed to the Proceedings of the $6^{\text {th }}$ International Conference on Hyperons, Charm and Beauty Hadrons

6/27/2004-7/03/2004, Chicago, USA

Stanford Linear Accelerator Center, Stanford University, Stanford, CA 94309

Work supported in part by Department of Energy contract DE-AC03-76SF00515. 


\section{Introduction}

The term "rare" B decays refers to B decays with small branching fractions (BF), usually less than $10^{-5}$. These are $\mathrm{B}$ decays that do not involve $b \rightarrow c$ transitions that are favoured by a large Cabibbo-Kobayashi-Maskawa (CKM) matrix element. They proceed, typically, through tree $b \rightarrow u$ transitions that are suppressed by a small CKM matrix element and/or through second order (penguin) $b \rightarrow s$ or $b \rightarrow d$ transitions. When both tree and penguin transitions are present, the interference between their amplitudes might be significant, leading to direct CP violation.

The direct $\mathrm{CP}$ violation in $B$ decays occurs when the decay rate of a $B$ into a final state is different than the decay rate of its antiparticle in the charge-conjugate final state. The difference is quantified by the time-integrated $\mathrm{CP}$ asymmetry $\left(A_{C P}\right)$ defined as the difference of the two decay rates normalised to their sum.

Rare $B$ decays into hadronic final states are of interest for both Standard Model and new physics studies.

Precise tests of the Standard Model predictions, including tests of the CP violation mechanism, can be achieved with these small amplitude processes. Many of these processes are potential tools for extracting the angles of the CKM Unitarity Triangle, providing constraints on the CKM parameters.

The penguin transitions that are expected to be present in many of the rare hadronic $\mathrm{B}$ decays might be a source of new physics as new particles, not predicted by the Standard Model, can be present in the loop transitions. Insights into the new physics can be learned from these rare decays.

The field of rare B decays has expended rapidly with the operation of the new $B$ factories, the asymmetric-energy $e^{+} e^{-}$colliders PEP-II at SLAC and KEKB at KEK. Each one produced more than 100million $B \bar{B}$ pairs allowing the BABAR and Belle experiments to make the observation of new decay channels or precise measurements of channels previously observed, mostly by the CLEO experiment.

Due to space restrictions, this review covers only a limited number of $B_{u}$ and $B_{d}$ (herein referred to as $B$ ) decay channels into mesonic final states, namely: $B \rightarrow \pi \pi, K \pi, K K, B \rightarrow\left(\eta \eta^{\prime}\right)\left(K, K^{*}, \rho, \pi\right)$, $B \rightarrow \rho K, \rho \pi$ and $B \rightarrow \phi K^{*}, \rho \rho, \rho K^{*}$.

The experimental values of $\mathrm{BF}$ and $A_{C P}$, as measured by the BABAR, Belle and CLEO experiments at the time of this conference (July 2004), as well as the average values calculated by the Heavy Flavor Averaging Group (HFAG) [1] are presented.

\section{$2 \quad B \rightarrow \pi \pi, K \pi, K K$ decays}

Two-body decays of $B$ to combinations of pions and/or kaons provide information that can be used to determine the angles $\alpha$ and $\gamma\left(\phi_{2}\right.$ and $\phi_{3}$ in another common notation) of the CKM Unitarity Triangle. Precise measurements of their BFs allow us to set limits on the theoretical hadronic uncertainties that affect the extraction of these angles.

The decay amplitudes of these processes have contributions from tree and/or penguin amplitudes. The contribution of the penguin amplitude can be determined from BF measurements and the direct $\mathrm{CP}$ violation can be established with $A_{C P}$ measurements.

The current BF measurements for these two-body decays are summarised in Table 1.

$B \rightarrow \pi^{0} \pi^{0}$ decay mode was recently observed by the BABAR and Belle experiments with a statistical significance of 4.2 and 3.4 standard deviations, respectively. The measured BFs are 
Table 1: BF $\left(\times 10^{-6}\right)$ of the $\pi \pi, K \pi$ and $K K$ decay modes.

\begin{tabular}{llllllll}
\hline Mode & BABAR & Ref. & Belle & Ref. & CLEO & Ref. & Average \\
\hline$\pi^{+} \pi^{-}$ & $4.7 \pm 0.6 \pm 0.2$ & {$[21]$} & $4.4 \pm 0.6 \pm 0.3$ & {$[28]$} & $4.5_{-1.2-0.4}^{+1.4+0.5}$ & {$[40]$} & $4.6 \pm 0.4$ \\
$\pi^{+} \pi^{0}$ & $5.5_{-0.9}^{+1.0} \pm 0.6$ & {$[26]$} & $5.0 \pm 1.2 \pm 0.5$ & {$[28]$} & $4.6_{-1.6-0.7}^{+1.8+0.6}$ & {$[40]$} & $5.2 \pm 0.8$ \\
$\pi^{0} \pi^{0}$ & $2.1 \pm 0.6 \pm 0.3$ & {$[22]$} & $1.7 \pm 0.6 \pm 0.2$ & {$[32]$} & $<4.4$ & {$[40]$} & $1.9 \pm 0.5$ \\
\hline$K^{+} \pi^{-}$ & $17.9 \pm 0.9 \pm 0.7$ & {$[21]$} & $18.5 \pm 1.0 \pm 0.7$ & {$[28]$} & $18.0_{-2.1-0.9}^{+2.3+1.2}$ & {$[40]$} & $18.2 \pm 0.8$ \\
$K^{+} \pi^{0}$ & $12.8_{-1.1}^{+1.2} \pm 1.0$ & {$[26]$} & $12.0 \pm 1.3_{-0.9}^{+1.3}$ & {$[28]$} & $12.90_{-2.2-1.1}^{+2.4+1.2}$ & {$[40]$} & $12.5_{-1.0}^{+1.1}$ \\
$K^{0} \pi^{+}$ & $22.3 \pm 1.7 \pm 1.1$ & {$[15]$} & $22.0 \pm 1.9 \pm 1.1$ & {$[28]$} & $18.8_{-3.3-1.8}^{+3.7+2.1}$ & {$[40]$} & $21.8 \pm 1.4$ \\
$K^{0} \pi^{0}$ & $11.4 \pm 1.7 \pm 0.8$ & {$[15]$} & $11.7 \pm 2.3_{-1.3}^{+1.2}$ & {$[28]$} & $12.8_{-3.3-1.4}^{+4.0+1.7}$ & {$[40]$} & $11.7 \pm 1.4$ \\
\hline$K^{+} K^{-}$ & $<0.6$ & {$[21]$} & $<0.7$ & {$[28]$} & $<0.8$ & {$[40]$} & $<0.6$ \\
$K^{+} \bar{K}^{0}$ & $<2.5$ & {$[15]$} & $<3.3$ & {$[28]$} & $<0.7$ & {$[40]$} & $<0.7$ \\
$K^{0} \bar{K}^{0}$ & $<1.8$ & {$[15]$} & $<1.5$ & {$[28]$} & $<3.3$ & {$[40]$} & $<1.5$ \\
\hline
\end{tabular}

Table 2: $A_{C P}$ of the $\pi \pi, K \pi$ and $K K$ decay modes.

\begin{tabular}{lccc}
\hline Mode & \multicolumn{1}{c}{ RABAR } & \multicolumn{1}{c}{ Ref. CLEO } & Ref. Average \\
\hline$\pi^{+} \pi^{-}$ & $+0.53 \pm 0.19$ & {$[5]$} & $+0.53 \pm 0.19$ \\
$\pi^{+} \pi^{0}-0.03_{-0.17}^{+0.18} \pm 0.02$ & {$[26]+0.00 \pm 0.10 \pm 0.02$} & {$[49]$} & $-0.01 \pm 0.09$ \\
\hline$K^{+} \pi^{-}-0.107 \pm 0.041 \pm 0.013[45]-0.088 \pm 0.035 \pm 0.018[45]$ & $-0.04 \pm 0.16$ & {$[47]-0.095 \pm 0.028$} \\
$K^{+} \pi^{0}-0.09 \pm 0.09 \pm 0.01$ & {$[26]+0.06 \pm 0.06 \pm 0.02$} & {$[49]-0.29 \pm 0.23 \pm 0.02[47]$} & $-0.00 \pm 0.05$ \\
$K^{0} \pi^{+}-0.05 \pm 0.08 \pm 0.01$ & {$[15]+0.05 \pm 0.05 \pm 0.01$} & {$[17]+0.18 \pm 0.24 \pm 0.02[47]+0.03 \pm 0.04$} \\
$K^{0} \pi^{0}+0.03 \pm 0.36 \pm 0.11$ & {$[15]+0.16 \pm 0.29 \pm 0.05$} & {$[49]$} & $+0.11 \pm 0.23$ \\
\hline
\end{tabular}

higher than the theoretical predictions of less than $10^{-6}[2]$.

The BFs of $K^{+} \pi^{-, 0}$ decay modes were found by all three experiments to be much higher than the BFs of $\pi^{+} \pi^{-, 0}$ decay modes. As the former modes are dominated by penguin transitions, this experimental result demonstrates that the penguin amplitude is significant.

The measured BFs of $\pi^{+} \pi^{-}$and $\pi^{+} \pi^{0}$ decay modes indicate a ratio $2 \Gamma\left(\pi^{+} \pi^{0}\right) / \Gamma\left(\pi^{+} \pi^{-}\right) \simeq 2$, where $\Gamma$ represents the decay rate of $B$ into the specified final state. The theoretical calculations that assume the dominance of the tree amplitudes in both decays [3] predict a ratio equal to unity. This discrepancy indicates a significant penguin contribution to the $\pi^{+} \pi^{-}$decay amplitude, the so called "penguin pollution".

$B \rightarrow K K$ decays are modes where the rescattering effects are expected to be significant. The available data only set upper limits on their BFs. Their comparison with perturbative QCD predictions [4] does not yet indicate evidence for rescattering effects.

The current $A_{C P}$ measurements of the specified two-body decays are presented in Table 2 .

Significant direct CP violation was reported by the Belle collaboration for the $B \rightarrow \pi^{+} \pi^{-}$ decay mode. The report was based on a time-dependent analysis [5] that is not discussed here. A preliminary time-integrated CP asymmetry measurement was also reported: $+0.53 \pm 0.19$.

No other significant direct CP asymmetry was reported for these decays. It is worth observing, however, that both BABAR and Belle experiments measured a non-zero asymmetry in the $K^{+} \pi^{-}$ decay mode with a statistical significance higher than two standard deviations. 
Table 3: $\mathrm{BF}\left(\times 10^{-6}\right)$ of the $(\eta, \eta \prime)\left(K, K^{*}, \rho, \pi\right)$ decay modes.

\begin{tabular}{llllllll}
\hline Mode & BABAR & Ref. & Belle & Ref. & CLEO & Ref. & Average \\
\hline$\eta \prime K^{+}$ & $76.9 \pm 3.5 \pm 4.4$ & {$[16]$} & $78 \pm 6 \pm 9$ & {$[29]$} & $80_{-0.9}^{+10} \pm 7$ & {$[41]$} & $77.6_{-4.5}^{+4.6}$ \\
$\eta K^{+}$ & $3.4 \pm 0.8 \pm 0.2$ & {$[24]$} & $5.3_{-1.5}^{+1.8} \pm 0.6$ & {$[31]$} & $2.2_{-2.2}^{+2.8}$ & {$[41]$} & $3.7 \pm 0.7$ \\
$\eta \prime K^{0}$ & $60.6 \pm 5.6 \pm 4.6$ & {$[16]$} & $68 \pm 10_{-8}^{+9}$ & {$[29]$} & $89_{-16}^{+18} \pm 9$ & {$[41]$} & $65.2_{-5.9}^{+6.0}$ \\
$\eta K^{0}$ & $<5.2$ & {$[24]$} & & & $<9.3$ & {$[41]$} & $<5.2$ \\
\hline$\eta \prime K^{*+}$ & $<14$ & {$[17]$} & $<90$ & {$[29]$} & $<35$ & {$[41]$} & $<14$ \\
$\eta K^{*+}$ & $25.6 \pm 4.0 \pm 2.5$ & {$[17]$} & $26.5_{-7.0}^{+7.8} \pm 3.0$ & {$[29]$} & $26.4_{-8.2}^{+9.6} \pm 3.3$ & {$[41]$} & $25.9_{-3.6}^{+3.8}$ \\
$\eta \prime K^{* 0}$ & $<7.6$ & {$[17]$} & $<20$ & {$[29]$} & $<24$ & {$[41]$} & $<7.6$ \\
$\eta K^{* 0}$ & $18.6 \pm 2.3 \pm 1.2$ & {$[17]$} & $16.5_{-4.2}^{+4.6} \pm 1.2$ & {$[29]$} & $13.8_{-4.6}^{+5.5} \pm 1.6$ & {$[41]$} & $17.5_{-2.1}^{+2.2}$ \\
\hline$\eta \rho^{+}$ & $<14$ & {$[17]$} & $<6.2$ & {$[29]$} & $<15$ & {$[41]$} & $<6.2$ \\
$\eta \rho^{0}$ & $<1.5$ & {$[17]$} & $<5.5$ & {$[31]$} & $<10$ & {$[41]$} & $<1.5$ \\
$\eta \pi^{+}$ & $5.3 \pm 1.0 \pm 0.3$ & {$[24]$} & $5.7_{-1.7}^{+1.4} \pm 0.9$ & {$[31]$} & $1.2_{-1.2}^{+2.8}$ & {$[41]$} & $4.9_{-0.8}^{+0.9}$ \\
$\eta \pi^{0}$ & $<2.5$ & {$[17]$} & & & $<2.9$ & {$[41]$} & $<2.5$ \\
\hline$\eta \prime \rho^{+}$ & $<22$ & {$[17]$} & & & $<33$ & {$[41]$} & $<22$ \\
$\eta \prime \rho^{0}$ & $<4.3$ & {$[17]$} & $<14$ & {$[29]$} & $<12$ & {$[41]$} & $<4.3$ \\
$\eta \prime \pi^{+}$ & $<4.5$ & {$[24]$} & $<7$ & {$[30]$} & $<12$ & {$[41]$} & $<4.5$ \\
$\eta \prime \pi^{0}$ & $<3.7$ & {$[17]$} & & & $<5.7$ & {$[41]$} & $<3.7$ \\
\hline
\end{tabular}

Table 4: $A_{C P}$ of the $\left(\eta, \eta^{\prime}\right)\left(K, K^{*}, \rho, \pi\right)$ decay modes.

\begin{tabular}{lll}
\hline Mode & BABAR & Ref. \\
\hline$\eta \prime K^{+}$ & $+0.04 \pm 0.05 \pm 0.01$ & {$[16]$} \\
$\eta K^{+}$ & $-0.52 \pm 0.24 \pm 0.01$ & {$[24]$} \\
\hline$\eta K^{*+}$ & $+0.13 \pm 0.14 \pm 0.02$ & {$[17]$} \\
$\eta K^{* 0}$ & $-0.02 \pm+0.11 \pm 0.02$ & {$[17]$} \\
\hline$\eta \pi^{+}$ & $-0.44 \pm 0.18 \pm 0.01$ & {$[24]$} \\
$\eta \rho^{+}$ & $+0.06 \pm 0.29 \pm 0.02$ & {$[17]$} \\
\hline
\end{tabular}

\section{$3 \quad B \rightarrow\left(\eta, \eta^{\prime}\right)\left(K, K^{*}, \rho, \pi\right)$ decays}

$B$ decays to final states containing an $\eta$ or an $\eta^{\prime}$ provide useful information for understanding the relative contribution of the tree and penguin amplitudes and have potential for establishing the direct CP violation in the Standard Model.

The decay amplitudes of $B \rightarrow(\eta, \eta \prime)\left(K^{+}, K^{*+}\right)$ have contributions from a CKM suppressed tree $b \rightarrow u$ amplitude and from two $b \rightarrow s$ penguin amplitudes that suffer a destructive interference [6]. An additional contribution of a flavor-singlet penguin amplitude to the $\eta / K^{*+}$ decay amplitude is expected [7]. The interference between the two penguin amplitudes and the $\eta / \eta^{\prime}$ mixing angle make the $\eta \prime K^{+}$and $\eta K^{*+}$ decay modes to be significantly enhanced relative to $\eta K^{+}$and $\eta \prime K^{*+}$ decay modes, respectively. A similar mechanism is expected for $K^{0}$ and $K^{* 0}$ decay modes except there is no tree amplitude contribution.

The experimental BF measurements, summarized in Table 3, confirm the predicted relationship between the BFs of these decays. Their absolute values are in agreement with recent next-toleading-order QCD calculations [8]. 
Table 5: $\mathrm{BF}\left(\times 10^{-6}\right)$ of the $\rho \pi$ and $\rho K$ decay modes.

\begin{tabular}{llllllll}
\hline Mode & BABAR & Ref. & Belle & Ref. & CLEO & Ref. & Average \\
\hline$\rho^{+} \pi^{-}$ & $22.6 \pm 1.8 \pm 2.2$ & {$[18]$} & $29.1_{-4.9}^{+5.0} \pm 4.0$ & {$[33]$} & $27.6_{-7.4}^{+8.4} \pm 4.2$ & {$[43]$} & $24.0 \pm 2.5$ \\
$\rho^{+} \pi^{0}$ & $10.9 \pm 1.9 \pm 1.9$ & {$[19]$} & $13.2 \pm 2.3_{-1.9}^{+1.4}$ & {$[39]$} & $<43$ & {$[43]$} & $12.0 \pm 1.9$ \\
$\rho^{0} \pi^{+}$ & $9.5 \pm 1.1 \pm 0.8$ & {$[19]$} & $8.0_{-2.0}^{+2.3} \pm 0.7$ & {$[35]$} & $10.4_{-3.4}^{+3.3} \pm 2.1$ & {$[43]$} & $9.2_{-1.1}^{+1.2}$ \\
$\rho^{0} \pi^{0}$ & $<2.9$ & {$[19]$} & $5.1 \pm 1.6 \pm 0.9$ & {$[5]$} & $<5.5^{+3} \pm$ & {$[43]$} & $5.1 \pm 1.6 \pm 0.9$ \\
\hline$\rho^{-} K^{+}$ & $7.3_{-1.2}^{+1.3} \pm 1.3$ & {$[18]$} & $15.1_{-3.3-2.6}^{+3.4+2.4}$ & {$[37]$} & $16_{-6}^{+8} \pm 3$ & {$[43]$} & $9.0 \pm 1.6$ \\
$\rho^{0} K^{+}$ & $3.9 \pm 1.2_{-3.5}^{+1.3}$ & {$[20]$} & $3.9^{+} \pm 0.6_{-0.4}^{+0.8}$ & {$[38]$} & $8.4_{-3.4}^{+4.0} \pm 1.8$ & {$[43]$} & $4.1_{-0.7}^{+0.9}$ \\
\hline
\end{tabular}

Table 6: $A_{C P}$ of the $\rho \pi$ and $\rho K$ decay modes.

\begin{tabular}{llllll}
\hline Mode & BABAR & Ref. & Belle & Ref. & Average \\
\hline$\rho^{+} \pi^{-}$ & $-0.11 \pm 0.06 \pm 0.03$ & {$[45]$} & $-0.38_{-0.21-0.05}^{+0.19+0.04}$ & {$[37]$} & $-0.14 \pm 0.06$ \\
$\rho^{+} \pi^{0}$ & $+0.24 \pm 0.16 \pm 0.06$ & {$[46]$} & $+0.06 \pm 0.19+0.04$ & {$[51]$} & $0.16 \pm 0.13$ \\
$\rho^{0} \pi^{+}$ & $-0.19 \pm 0.11 \pm 0.02$ & {$[46]$} & & & $-0.19 \pm 0.11$ \\
\hline$\rho^{-} K^{+}$ & $+0.18 \pm 0.12 \pm 0.08$ & {$[45]$} & $+0.22_{-0.23-0.02}^{+0.22+0.06}$ & {$[37]$} & $+0.19 \pm 0.12$ \\
\hline
\end{tabular}

$B \rightarrow(\eta, \eta \prime)(\rho, \pi)$ decay amplitudes are dominated by an external tree $b \rightarrow u$ amplitude while an internal tree $b \rightarrow u$ amplitude is color suppressed and two penguin $b \rightarrow s$ amplitudes are CKM suppressed. The interference between the two penguin amplitudes is reduced while the interference between the tree and penguin ones is significant, enhancing the $\eta^{(\prime)} \rho^{+}$decay modes.

The present amount of experimental data provided only upper limits for the BFs of most of these decay modes. They are presented in Table 3. It can be observed that the upper limit of $\eta^{\prime} \rho^{+}$ $\mathrm{BF}$ is much higher than of the other modes.

The destructive interference between the two penguin amplitudes of the $\eta^{(\prime)} K^{(*)}$ decay modes make the interference between the CKM suppressed tree amplitude and the penguin amplitudes significant for the suppressed modes, leading to large $A_{C P}$. Large $A_{C P}$ are expected for $\eta \pi$ decay mode as well.

Preliminary $A_{C P}$ measurements are reported by the BABAR collaboration for six decay modes. They are shown in Table 4 . The asymmetry for $\eta / K^{*}$ decay mode was also measured by the Belle $(-0.02 \pm 0.07 \pm 0.01[48])$ and CLEO $(+0.03 \pm 0.12 \pm 0.02[47])$ experiments.

While no significant CP asymmetry can be concluded from these measurements, the results for $\eta K^{+}$and $\eta \pi^{+}$can be noted as being non-zero with a statistical significance higher than two standard deviations.

\section{$4 \quad B \rightarrow \rho \pi, \rho K$ decays}

$B$ decaying to $\rho \pi$ and $\rho K$ final states are important decay channels for constraining the CKM parameters and for testing the theoretical models that predict the BFs in a quite large range.

The isospin analysis of the $\rho \pi$ decay amplitudes is one of the main method for determining the angle $\alpha$ of the CKM Unitarity Triangle. The decay amplitudes are extracted from the BF and $A_{C P}$ experimental values.

The current BF measurements of these decays are shown in Table 5.

The $\rho^{0} \pi^{0}$ decay mode, very much needed for the above mentioned isospin analysis, was recently 
Table 7: $\mathrm{BF}\left(\times 10^{-6}\right)$ of the $\phi K^{*}, \rho \rho$ and $\rho K^{*}$ decay modes.

\begin{tabular}{llllllll}
\hline Mode & BABAR & Ref. & Belle & Ref. & CLEO & Ref. & Average \\
\hline$\Phi K^{*+}$ & $12.7_{-2.0}^{+2.2} \pm 1.1$ & {$[23]$} & $6.7_{-1.9-1.0}^{+2.1+0.7}$ & {$[36]$} & $10.6_{-4.9-1.6}^{+6.4+1.8}$ & {$[42]$} & $9.7 \pm 1.5$ \\
$\Phi K^{* 0}$ & $11.2 \pm 1.3 \pm 0.8$ & {$[23]$} & $10.0_{-1.5-0.8}^{+1.60 .7}$ & {$[36]$} & $11.5_{-3.7-1.7}^{+4.5+1.8}$ & {$[42]$} & $10.7 \pm 1.1$ \\
\hline$K^{*+} \rho^{0}$ & $10.6_{-2.6}^{+3.0} \pm 2.4$ & {$[23]$} & & & $<74$ & {$[44]$} & $10.6_{-3.5}^{+3.8}$ \\
\hline$\rho^{+} \rho^{-}$ & $30 \pm 4 \pm 5$ & {$[27]$} & & & & $30 \pm 6$ \\
$\rho^{+} \rho^{0}$ & $22.5_{-5.4}^{+5.7} \pm 5.8$ & {$[23]$} & $31.7 \pm 7.1_{-6.7}^{+3.8}$ & {$[34]$} & & & $26.4_{-6.4}^{+6.1}$ \\
$\rho^{0} \rho^{0}$ & $<2.1$ & {$[23]$} & & & $<18$ & {$[44]$} & $<2.1$ \\
\hline
\end{tabular}

Table 8: $A_{C P}$ of the $\phi K^{*}, \rho \rho, \rho K^{*}$ decay modes.

\begin{tabular}{llllll}
\hline Mode & BABAR & Ref. & Belle & Ref. & Average \\
\hline$\Phi K^{*+}$ & $+0.16 \pm 0.17 \pm 0.03$ & {$[23]$} & $-0.13 \pm 0.29_{-0.11}^{+0.08}$ & {$[50]$} & $+0.15 \pm 0.15$ \\
$\Phi K^{* 0}$ & $+0.04 \pm 0.12 \pm 0.02$ & {$[23]$} & $+0.07 \pm 0.15_{-0.03}^{+0.05}$ & {$[50]$} & $+0.05 \pm 0.10$ \\
\hline$K^{*+} \rho^{0}$ & $+0.20_{-0.29}^{+0.32} \pm 0.04$ & {$[23]$} & & & $+0.20_{-0.29}^{+0.32}$ \\
\hline$\rho^{+} \rho^{0}$ & $-0.19 \pm 0.23 \pm 0.03$ & {$[23]$} & $+0.00 \pm 0.22 \pm 0.03$ & {$[25]$} & $-0.09 \pm 0.16$ \\
\hline
\end{tabular}

measured by the Belle experiment with a statistical significance of 3.5 standard deviations. The measured $\mathrm{BF}$ is higher than most of the theoretical predictions, suggesting that some contributions to the decay amplitude might be higher than expected, making the isospin analysis more complicated.

$A_{C P}$ was measured for four decay modes and the results are shown in Table 6. No asymmetry is observed in these decays.

\section{$5 \quad B \rightarrow \phi K^{*}, \rho \rho, \rho K^{*}$ decays}

$B$ decays to vector-vector final states are of particular interest as more observables are available for $\mathrm{CP}$ violation tests: the angular distributions and the vector meson polarization components (as well as $\mathrm{BF}$ and $\left.A_{C P}\right)$. In addition, $\rho \rho$ decay modes, being very similar to $\rho \pi$ modes, provide an alternative method for extracting the angle $\alpha$ of the CKM Unitarity Triangle.

$B \rightarrow \Phi K^{*}$ decay proceeds through a pure $b \rightarrow s$ penguin transition offering the possibility of an unambiguous signature for penguins. As the penguin transitions are the same for $\Phi K^{* 0}$ and $\Phi K^{*+}$ decay modes, the difference being only the spectator quark, similar BFs are expected. Indeed, the measurements performed by the three experiments, summarized in Table 7, confirm these expectations.

$B \rightarrow \rho \rho$ modes proceed through $b \rightarrow u$ tree and CKM suppressed $b \rightarrow d$ penguin transitions. Their measured BFs are also shown in Table 7. While both BABAR and Belle experiments observed the $\rho^{+} \rho^{0}$ decay mode, BABAR collaboration also reported the observation of the $\rho^{+} \rho^{-}$decay mode with a statistical significance of 5.1 standard deviations and lowered the limit on $\rho^{0} \rho^{0} \mathrm{BF}$.

The $A_{C P}$ is expected to be large for $\rho K^{*}$ decay modes that proceed through both $b \rightarrow u$ tree and $b \rightarrow s$ penguin transitions. This asymmetry is expected to be small in the Standard Model and sizable in the presence of new physics effects in the other decay channels.

The current $A_{C P}$ measurements, summarized in Table 8, do not indicate significant CP asym- 
Table 9: The longitudinal polarization fraction of the $\phi K^{*}, \rho \rho, \rho K^{*}$ decay modes.

\begin{tabular}{lll}
\hline Mode & BABAR & Ref. \\
\hline$\Phi K^{*+}$ & $0.46 \pm 0.12 \pm 0.03$ & {$[14]$} \\
$\Phi K^{* 0}$ & $0.52 \pm 0.07 \pm 0.02$ & {$[13]$} \\
\hline$K^{*+} \rho^{0}$ & $0.96_{-0.15}^{+0.04} \pm 0.04$ & {$[14]$} \\
\hline$\rho^{+} \rho^{-}$ & $0.99 \pm 0.03_{-0.03}^{0.04}$ & {$[14]$} \\
$\rho^{+} \rho^{0}$ & $0.97_{-0.07}^{+0.03} \pm 0.04$ & {$[14]$} \\
\hline
\end{tabular}

metry in these decays.

In the framework of the perturbative QCD [9], the longitudinal polarization fraction, $f_{L}=\frac{\Gamma_{L}}{\Gamma}$, is predicted to be very close to $100 \%$ for all vector-vector decay modes. This prediction is only partially confirmed by the present measurements. The values measured by the BABAR experiment, presented in Table 9, indicate $f_{L}$ close to $100 \%$ for the $\rho K^{*}$ and $\rho \rho$ decay modes and around $50 \%$ for the $\phi K^{*}$ decay modes. Similar results were obtained by the Belle experiment for the $\rho^{+} \rho^{0}$ mode $(0.948 \pm 0.106 \pm 0.021[12])$ and the $\phi K^{* 0}$ mode $(0.43 \pm 0.19 \pm 0.04$ [11]). Different theoretical interpretations exist for this result, some of them suggesting new physics effects in the $b \rightarrow s$ penguin transition [10].

\section{Conclusions}

The already impressive amount of data accumulated by the new B factories at SLAC and KEK yielded important advances in the field of rare hadronic B decays. A few of them are summarized here.

Precise measurements of $\mathrm{BF}$ and $A_{C P}$ were performed for many decay channels and new decay modes were observed. Two of these new modes are of particular importance: $B \rightarrow \pi^{+} \pi^{-}$with a $\mathrm{BF}$ of $(1.9 \pm 0.5) \cdot 10^{-6}$ and $B \rightarrow \rho^{0} \pi^{0}$ with a $\mathrm{BF}$ of $(5.1 \pm 1.6 \pm 0.9) \cdot 10^{-6}$.

The observation of direct $\mathrm{CP}$ violation in the $B \rightarrow \pi^{+} \pi^{-}$decay mode, in a time-dependent analysis, confirmed through a preliminary time-integrated $\mathrm{CP}$ asymmetry measurement of $+0.53 \pm$ 0.19, was reported by the Belle collaboration.

Non-zero direct CP asymmetries were measured for $K^{+} \pi^{-}, \eta K^{+}$and $\eta \pi^{+}$decay modes with statistical significances higher than two standard deviations. Updates of these measurements are eagerly expected.

The longitudinal polarization fraction of vector-vector decay modes was experimentally found close to $100 \%$ for $\rho \rho$ and $\rho K^{*}$ modes, confirming the theoretical predictions, and around $50 \%$ for $\phi K^{*}$ modes, much lower than the theoretical predictions. Theoretical efforts are needed in order to understand this discrepancy. 


\section{References}

[1] http://www.slac.stanford.edu/xorg/hfag

[2] M. Beneke et.al., Nucl. Phys. B606 (2001) 245, Y.Y. Keum, A. I. Sanda,hep-ph/0306004

[3] M. Gronau et. al, Phys. Rev. D65 (2002) 013004

[4] C. Chen et. al., Phs.Rev. D63 (2000) 014003

[5] Belle Coll., Phys. Rev. Lett. 93 (2004)

[6] H. J. Lipkin, Phys. Lett. B254 (1991) 247

[7] M. Beneke et. al., Nucl. Phys.B675 (2003) 333; C. Chiang et. al., Phys. Rev. D69 (2004) 034001

[8] M. Beneke et. al., Nucl. Phys. B651 (2003) 225

[9] M. Suzuki, Phys. Rev. D66 (2002) 051801

[10] Y. Grossman, Int. J. Mod. Phys., A19 (2004) 907

[11] Belle Coll., Phys. Rev. Lett. 91 (2003) 201801

[12] Belle Coll., Phys. Rev. Lett. 91 (2003) 221801

[13] J. Smith,talk at 39th Rencontres de Moriond on QCD, La Thuile, Italy, 28 March 2004

[14] BABAR Coll., Phys.Rev.Lett. 91 (2003) 171802

[15] BABAR Coll., Phys.Rev.Lett. 92 (2004) 201802

[16] BABAR Coll., Phys.Rev.Lett. 91 (2003) 161801

[17] BABAR Coll., hep-ex/0403025

[18] BABAR Coll., Phys.Rev.Lett. 91 (2003) 201802

[19] BABAR Coll., Phys.Rev.Lett. 93 (2004) 051802

[20] BABAR Coll., hep-ex/0308065

[21] BABAR Coll., Phys.Rev.Lett. 89 (2001) 281802

[22] BABAR Coll., Phys.Rev.Lett. 91 (2003) 241801

[23] BABAR Coll., Phys.Rev.Lett. 91 (2003) 171802

[24] BABAR Coll., Phys.Rev.Lett. 92 (2004) 061801

[25] BABAR Coll., Phys.Rev. D69 (2004) 011102

[26] BABAR Coll., Phys.Rev.Lett. 91 (2003) 021801

[27] BABAR Coll., hep-ex/0404029 
[28] Belle Coll., Phys.Rev. D69 (2004) 111102

[29] H. Aihara, talk at Flavor Physics and CP Violation, Paris, France, 3 June 2003

[30] Belle Coll., Phys. Lett. B517 (2001) 309

[31] Belle Coll., hep-ex/0205062

[32] Belle Coll., Phys.Rev.Lett. 91 (2003) 261801

[33] Belle Coll., BELLE-CONF-0318 (2003)

[34] Belle Coll., Phys.Rev.Lett. 91, 221801 (2003)

[35] Belle Coll., Phys. Lett. B542 (2002) 183

[36] Belle Coll., Phys.Rev.Lett. 91 (2003) 201801

[37] Belle Coll., BELLE-CONF-0317 (2003)

[38] Belle Coll., BELLE-CONF-0338 (2003)

[39] J. Zhang, talk at 39th Rencontres de Moriond QCD, La Thuile, Italy, 28 March 2004

[40] CLEO Coll. Phys.Rev. D68 (2003) 052002

[41] CLEO Coll. Phys.Rev.Lett. 85 (2000) 520

[42] CLEO Coll. Phys.Rev.Lett. 86 (2001) 3718

[43] CLEO Coll. Phys.Rev.Lett. 85 (2000) 2881

[44] CLEO Coll. Phys.Rev.Lett. 88 (2002) 021802

[45] J. Fry, Int. J. Mod. Phys. A19 (2004) 918

[46] BABAR Coll., hep-ex/0307087

[47] CLEO Coll., Phys.Rev.Lett. 85 (2000) 525

[48] Belle Coll., Phys. Lett. B546 (2002) 196

[49] R. Stamen, talk at Lake Louis Winter Institute, Alberta, Canada, 15 Feb. 2004

[50] Belle Coll., hep-ex/0307014

[51] F. Ronga,talk at 39th Rencontres de Moriond on QCD, La Thuile,Italy, 28 March 2004 